\title{
Nearly 50 Years of Child Exposure to Intimate Partner Violence Empirical Research: Evidence Mapping, Overarching Themes, and Future Directions
}

\author{
Megan R. Holmes ${ }^{1} \cdot$ Kristen A. Berg $^{1,2} \cdot$ Anna E. Bender $^{1} \cdot$ Kylie E. Evans $^{1} \cdot$ Kari O'Donnell $^{1} \cdot$ Emily K. Miller $^{1}$
}

Published online: 12 January 2022

(c) The Author(s), under exclusive licence to Springer Science+Business Media, LLC, part of Springer Nature 2022

Research on children exposed to intimate partner violence (IPV) is approaching 50 years of study, with the first published case study detailing the negative impact of IPV exposure on children in 1975 (Levine, 1975) and the first wave of empirical publications appearing in the 1980s (Davis \& Carlson, 1987; Hughes \& Barad, 1983; Rosenbaum \& O'Leary, 1981; Stagg et al., 1989). During this nascent period, research focused primarily on symptom identification among IPV-exposed children. Evidence from early cross-sectional studies supported the anecdotal observations of clinicians, social workers, and advocates in the field: IPV exposure in children was linked to a higher risk of behavioral, emotional, social, and cognitive difficulties, and the impact of IPV on those outcomes varied based on a child's age and developmental stage (Evans et al., 2008; Fantuzzo \& Lindquist, 1989; Fantuzzo \& Mohr, 1999; Margolin, 1998; Osofsky, 1998). However, as the prominence of IPV exposure research grew, scholars increasingly called into question methodological themes pervasive in the literature and challenged the ability of existing research to draw causal process conclusions. Concerns included overreliance on shelter samples, potential underreporting of IPV victimization by mothers, lack of precision in defining and measuring IPV exposure, reliance on the Child Behavior Checklist (Achenbach \& Rescorla, 2001) to measure child outcomes, lack of theoretical grounding, and failure to account for cooccurring forms of maltreatment (Edleson, 1999; Fantuzzo

Megan R. Holmes

mholmes@case.edu

1 Center On Trauma and Adversity, Jack, Joseph and Morton Mandel School of Applied Social Sciences, Case Western Reserve University, 10900 Euclid Ave., Cleveland, OH 44106-7164, USA

2 Center for Health Care Research and Policy, The MetroHealth System, 2500 MetroHealth Dr., Cleveland, OH 44109, USA
\& Mohr, 1999; Holt et al., 2008; Margolin, 2005; Wolfe et al., 2003).

In the decades that followed, the rigor and complexity of IPV exposure studies grew exponentially. Moving beyond the identification of symptom profiles, research attention turned toward the contextual mediating and moderating mechanisms that influence child outcomes, including factors within the individual, family, neighborhood, school, and community environments (Evans et al., 2008; Fortin et al., 2011; Huang et al., 2010; O'Keefe, 1994; Owen et al., 2006). In an effort to address early methodological concerns, researchers increasingly utilized community samples, incorporated child reports of violence exposure, implemented longitudinal designs, and accounted for child polyvictimization. Theoretical models, including social learning theory, developmental psychopathology frameworks, trauma theory, and family systems theory, among others (Gewirtz \& Edleson, 2007; Graham-Bermann \& Hughes, 1999; Lichter \& McCloskey, 2004), emerged to guide empirical studies. Measurement precision grew as instruments were designed and validated to capture the unique and multifaceted experiences of children exposed to IPV, such as the Child Exposure to Domestic Violence (CEDV) scale (Edleson et al., 2008; Ravi \& Tonui, 2020). Importantly, resilience has emerged as a key interest in recent years. A heightened focus has been placed on identifying factors and mapping developmental trajectories associated with resilient functioning in IPV-exposed youth (Graham-Bermann et al., 2009; Holmes et al., 2018a, b; Hughes et al., 2001; Martinez-Torteya et al., 2009), thus aligning the field with the strengths-based framework that defines direct practice models.

The goal of this special section is to advance the scientific understanding of the effects of IPV exposure on child outcomes, provide future direction for research, and identify practice implications for IPV-exposed children. In this paper, we used an Evidence and Gap Map (EGM) 
to systematically identify and present all published quantitative evidence available through August 2020 from studies that examined the effect of IPV exposure on child outcomes. Using the EGM, seven paper topics were chosen for inclusion in this special section because, to our knowledge, no previous rigorous systematic review had been published on these topics, previous reviews on these topics were not systematic, or previous reviews focused on a narrow age range or developmental period. Holmes et al. (2021) synthesized research examining the relation between IPV exposure and medical system engagement and physical health outcomes, and investigated methodological factors that may influence these outcomes. Evans et al. (2021a) assessed empirical literature on adolescent dating violence (ADV) victimization and perpetration outcomes in youth exposed to IPV, the mechanisms that may explain ADV outcomes in IPV-exposed youth, and the methodological factors influencing interpretation of the findings. Bender et al. (2021) synthesized literature on the association between IPV exposure and children's social-emotional competence and the extent to which social-emotional competence played a mediating or moderating role between IPV exposure and other adjustment outcomes of IPV-exposed children. Lee et al. (2021) reviewed the literature examining the association between IPV exposure and bullying, as well as relevant environmental factors. Evans et al. (2021b) summarized research examining children's appraisals and acceptance of parental IPV. Berg et al. (accepted) investigated physiological correlates of IPV exposure and the risk and protective effects of children's bioregulatory systems in the context of IPV exposure. Cage et al. (2021) examined literature on the IPV-academic functioning relationship by reviewing outcome trends, critically assessing the strengths and limitations of the current body of knowledge and the extent to which this literature considered co-occurring risk factors.

\section{Method}

As shown in Fig. 1, we employed a staged approach for moving from the EGM to a narrative synthesis of the literature presented in this special section. Using procedures from the Campbell Collaboration (Saran \& White, 2018), an EGM was conducted to systematically identify and visually present all published quantitative research that examined the effect of IPV exposure on child outcomes. EGMs are developed using methods similar to systematic review by comprehensively and systematically searching and coding for evidence on a broad research topic. Due to the large scope of evidence mapped, critical appraisals of individual included studies are not often conducted; rather, EGMs provide a visual depiction of all relevant evidence (Saran \& White, 2018). The purpose of an EGM is to inform research priority-setting, identify gaps in the broad research area, and define the focus of future systematic reviews (Katz et al., 2003).

\section{EGM Method}

\section{Search Strategy}

In order to identify relevant published quantitative studies that examine the effect of IPV exposure on child outcomes, seven electronic bibliographic databases (CINAHL, ERIC, LexisNexis, Medline, PsychINFO, Social Science Citation Index, and Social Work Abstracts) were searched for articles published up through August 2020. LexisNexis was only searched up until 2016, and not from 2017 through 2020, as it did not yield any included studies that were not already captured by the other six databases up to 2016. Key search terms included "(domestic OR interparental OR family OR marital OR interpersonal) AND (expos* OR witness*) AND (violen* OR conflict OR abus* OR batter*) AND (child* OR youth)." Citations were then transferred into a bibliographic software management tool and deduplicated.

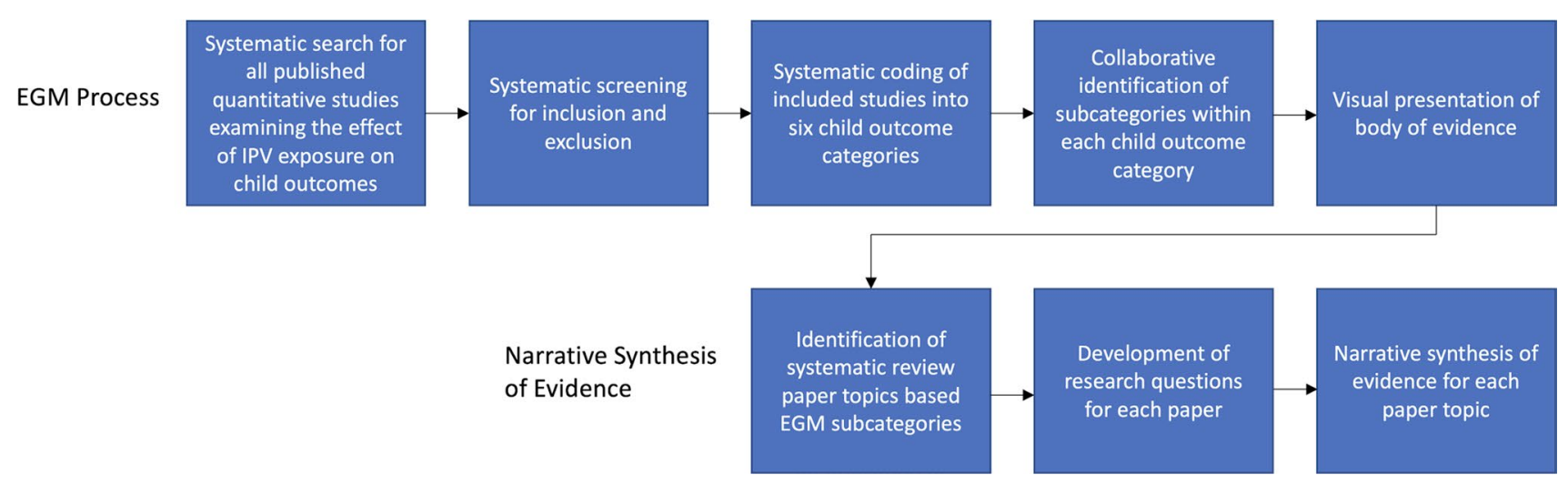

Fig. 1 Flow chart of procedures of moving from an Evidence and Gap Map (EGM) to a narrative synthesis of evidence 


\section{Inclusion and Exclusion Criteria}

Inclusion criteria were as follows: the study (1) was peerreviewed, published in English, and used quantitative methods; (2) contained participants ages birth through 17 years (or those with more than $80 \%$ of the sample ages under 18 to ensure majority of sample was within target age range); (3) measured IPV exposure compared with other children who had experienced another form of violence, polyvictimization, or who had never been exposed to violence; and (4) utilized validated or reliable scale with published psychometric data, administrative data, anthropometric data, biological outcome data, or measured the occurrence of a discrete and administratively verifiable event (e.g., hospitalization, being held back a grade in school) to measure child outcome variables. Articles were excluded if they were not peer-reviewed (e.g., dissertations), not available in English or full text, employed an adult retrospective report, only measured IPV exposure prenatally, and/or used qualitative methods, a case study, bivariate analyses with no multivariable analyses, or a descriptive design. In order to capture all published quantitative research, no limiters were set for the year of publication.

\section{Screening for Inclusion or Exclusion}

Two research assistants independently coded each citation for inclusion or exclusion based on a provided data extraction form. Abstracts were first reviewed and, when necessary, full-text articles were examined to determine inclusion or removal. Conflicting inclusion or exclusion decisions were reconciled by a third, doctoral-level research assistant. Citations were harvested from all review papers, deduplicated, and coded independently by two research assistants for inclusion. After the screening process was completed, full-text articles were comprehensively reviewed.

\section{Coding of Child Outcome Categories}

Research assistants completed the data extraction form for each included study, including details about the types of child outcomes evaluated, and the data were subsequently entered into a database. Two research assistants independently coded each included study into six child outcome categories: behavior problems (e.g., externalizing behaviors/behavior problems, aggression, delinquency, antisocial behavior), psychological outcomes (e.g., internalizing behaviors/symptoms, post-traumatic stress disorder or symptoms, anxiety symptoms/diagnosis, depression symptoms/diagnosis, emotional problems/dysregulation/insecurity), cognitive outcomes (e.g., acceptance of/appraisals of/beliefs about IPV, self-blame, academic functioning), social outcomes (e.g., social competence, bullying victimization or perpetration, peer relationships, dating violence victimization/perpetration), physical health outcomes (e.g., general health, lung functioning, BMI/obesity, medical engagement), and physiological outcomes (e.g., cortisol regulation, respiratory sinus arrhythmia/vagal regulation). Citations were assigned to more than one child outcome category if a study reported results for multiple outcomes. Conflicting child outcome category coding was reconciled by a third, doctoral-level research assistant. Research assistants then collaboratively identified the subcategories (e.g., academic functioning, general health) within each broader child outcome category according to outcomes' conceptual or operational similarity.

\section{Visual Presentation of Evidence}

Two figures were developed to curate and visually present the body of published quantitative literature on the effects of IPV exposure on child outcomes. First, we created a graph displaying yearly and cumulative publications. Second, we created a graph displaying the density of publications by child outcome categories and subcategories.

\section{Identification of Systematic Review Papers}

The EGM was used as the initial step in the planning of the systematic review papers. Based on findings from the EGM, we identified seven systematic review paper topics that had (a) a sufficient body of literature, and (b) either had no published systematic review on the topic, previous reviews on these topics were not systematic, or previous reviews focused on a narrow age range or developmental period. Next, our research team developed research questions for each paper topic. Finally, because rigorous systematic review methodology was used for the EGM to capture all published quantitative articles on the effect of IPV exposure on child outcomes, and rigorous screening and extracting methodology was used to assign included studies to child outcome categories and subcategories, a narrative synthesis of the evidence for each paper topic was completed.

\section{Results}

Searches from the seven databases yielded a total of 32,666 citations (see Fig. 2). After deduplication, 15,354 citations were identified for screening. Of those screened, 13,714 were excluded and the remaining 1,640 were screened for eligibility. Of the full-text articles, 1,258 did not meet eligibility criteria, resulting in a total of 381 citations included in the EGM. Research assistants coding the 15,354 screened citations agreed on 14,334 in terms of inclusion or exclusion (93.36\%). The remaining 1,072 
Fig. 2 Flow of information through each phase of the systematic review. Note. SSCI Social Science Citation Index, SWA Social Work Abstracts. Parenthetical study counts across categorized outcome domains are not mutually exclusive; an included study in one outcome domain could also be included, as relevant, in other outcome domains

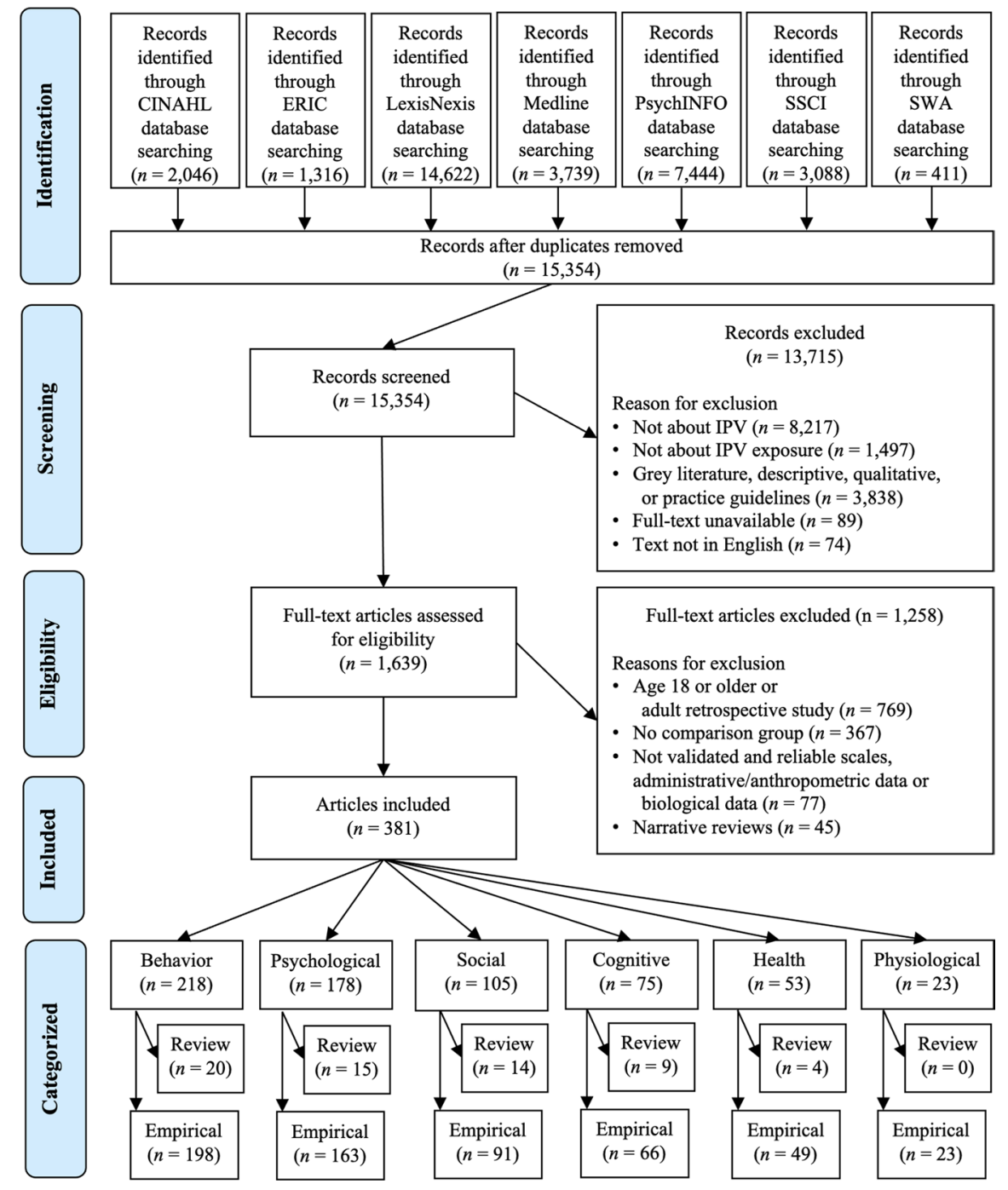

Note. SSCI $=$ Social Science Citation Index, SWA $=$ Social Work Abstracts. Parenthetical study

outcome domain could also be included, as relevant, in other outcome domains.

were screened by a third, doctoral-level research assistance for final determination of inclusion or exclusion.

Of the 381 included studies, 350 articles were empirical studies and 31 articles were review articles including eleven meta-analysis articles and twenty systematic review articles. The majority of articles $(n=218)$ examined the effect of IPV exposure on behavior outcomes (empirical studies, $n=198$; review articles, $n=20$ ), followed by 178 articles that examined child psychological outcomes (empirical studies, $n=163$; review articles, $n=15$ ), 105 articles that examined child social outcomes (empirical studies, $n=91$; review articles, $n=14), 75$ articles that examined child cognitive outcomes (empirical studies, $n=66$; review articles, $n=9$ ), 53 articles that examined child physical health outcomes (empirical studies, $n=49$; review articles, $n=4)$, and 23 articles that examined child physiological outcomes (empirical studies, $n=23$; review articles, $n=0$ ).

Included articles were published between 1982 and 2020 with review articles published between 1998 and 2020. Figure 3 shows the yearly and cumulative totals of published articles examining the effect of IPV exposure on child outcomes. In general, there was an increasing number of publications each year. Figure 4 displays the density of publications by child outcome categories and subcategories. The top of the figure displays density distributed across years by child outcome subcategories, the middle of the figure shows the total number of articles by child outcome subcategories, and the bottom of the figure shows the total number of review articles by child outcome subcategories. 


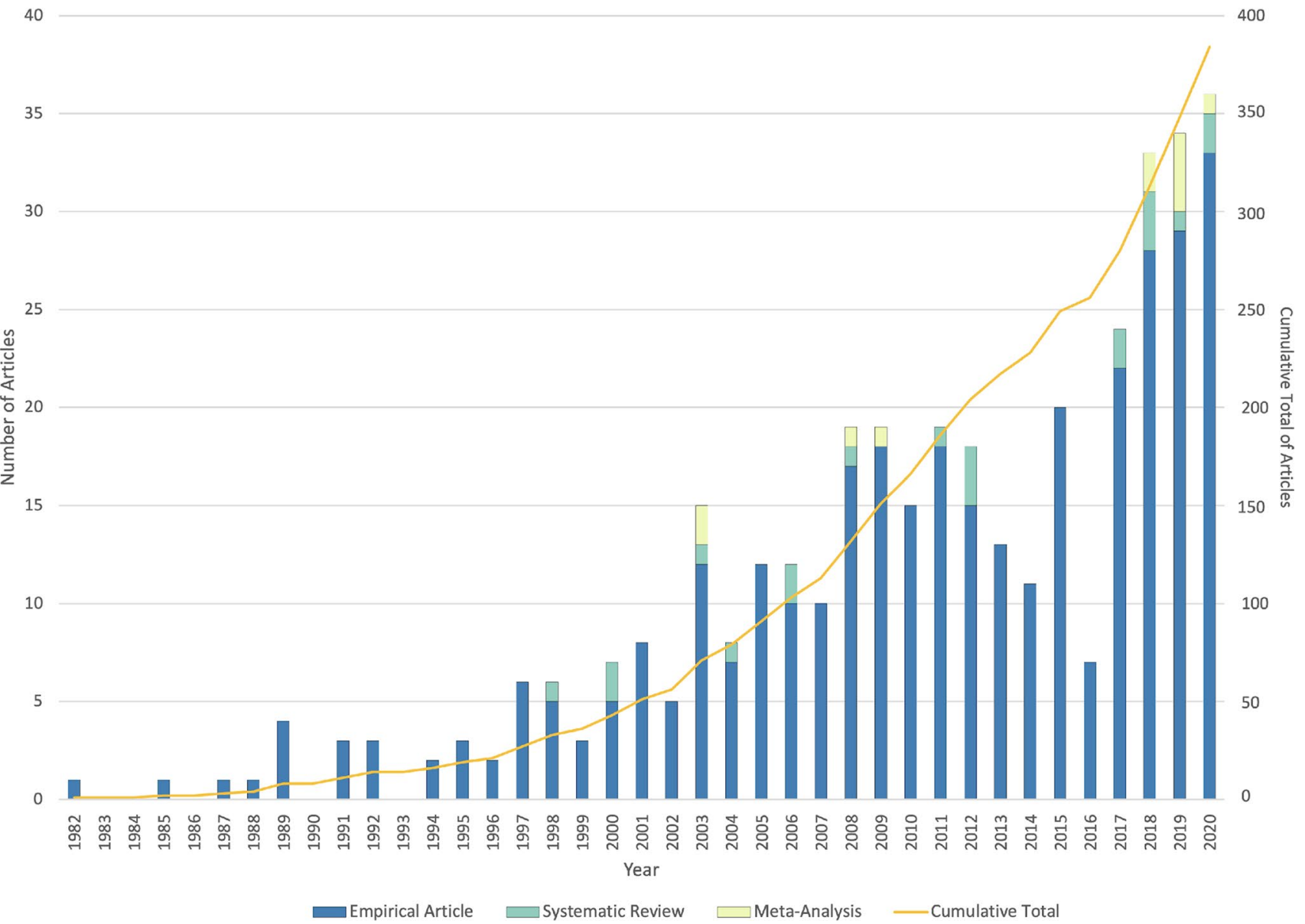

Fig. 3 Yearly and cumulative publications of the effects of IPV exposure on child outcomes

Based on the EGM findings displayed in Fig. 4, child outcome subcategories were reviewed by the research team and compared with published review articles (review articles are shown in Fig. 4 as yellow circles). Seven topics were identified for narrative synthesis and included in this special section: physical health and medical system engagement (31 articles; Holmes et al., 2021); adolescent dating violence victimization \& perpetration (29 articles; Evans et al., 2021a); social-emotional competence (26 articles; Bender et al., 2021); bullying victimization and perpetration (14 articles; Lee et al., 2021); IPV appraisal (13 articles; Evans et al., 2021b); physiological functioning (23 articles; Berg et al., accepted); and academic functioning (13 articles; Cage et al., 2021).

\section{Characteristics of Included Studies of Topic Papers}

\section{Physical Health and Medical Engagement}

Holmes et al. (2021) reviewed 31 articles that examined the physical health and medical system engagement of
IPV-exposed children, and the methodological considerations that contribute to the field's understanding of these relations. Regarding medical engagement, studies indicated that IPV-exposed children were less likely to be up to date on immunizations, and that IPV-exposed children experienced an increased rate of emergency room visits compared with non-exposed children. IPV exposure was also related to poorer lung functioning (i.e., asthma and respiratory problems), higher BMI score, and greater reported health concerns. Methodological considerations included study design, measurement of IPV exposure, measurement of medical engagement, and measurement of health outcomes.

\section{ADV Victimization and Perpetration}

Evans et al. (2021a) reviewed 29 articles focused on ADV outcomes among IPV-exposed youth. Findings were inconsistent regarding the link between IPV exposure and ADV perpetration and victimization. While several studies identified a significant relationship between IPV exposure and $A D V$ victimization or perpetration, nearly an equal number 


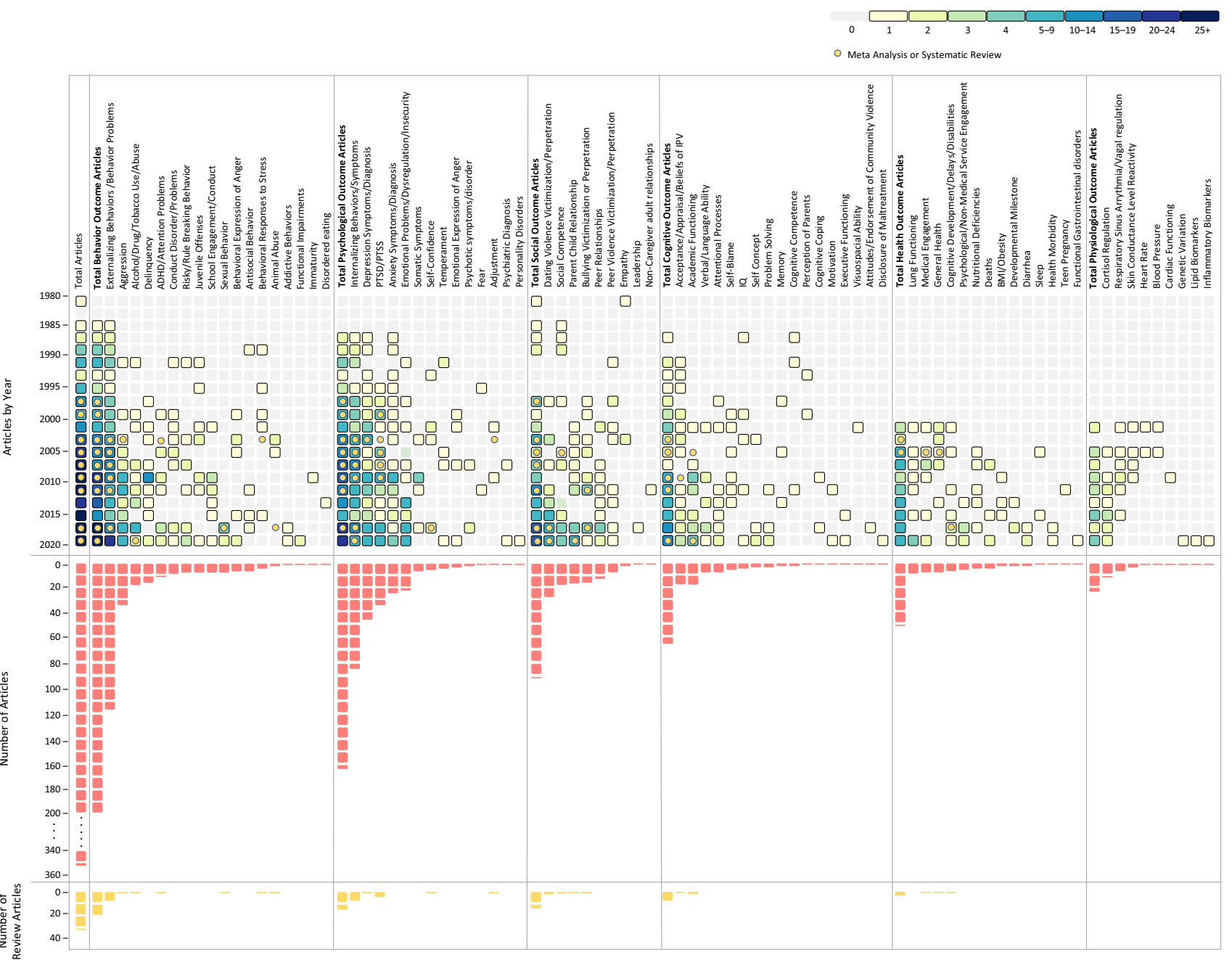

Note: The top of the figure displays density distributed across years by child outcome subcategories, the middle of the figure shows the total number of articles by child outcome subcategories, and the bottom of the figure shows the total number of review articles by child outcome subcategories.

Fig. 4 Evidence and Gap Map (EGM) of the effects of IPV exposure on child outcomes. Note: The top of the figure displays density distributed across years by child outcome subcategories, the middle of the figure shows the total number of articles by child outcome subcategories, and the bottom of the figure shows the total number of review articles by child outcome subcategories of studies did not, thus highlighting the multifinality of outcomes in this population. Findings were similarly mixed regarding mechanisms proposed to link IPV exposure and $\mathrm{ADV}$, including mental health symptoms, anger regulation, beliefs about the acceptability of violence, peer influences, and parenting practices. Research implications address the need for IPV-exposure studies on healthy relationship outcomes among this population, sexual and gender minority youth relationship patterns, and enhanced methodological rigor.

\section{Social-emotional Competence}

Bender et al. (2021) reviewed 26 articles that examined social-emotional competence outcomes among IPV-exposed children. Across the majority of studies, IPV-exposed youth demonstrated lower levels of social-emotional competence, and higher levels of emotional dysregulation compared with their non-exposed peers. Findings on the effects of IPV exposure on empathy development in children were more heterogeneous. However, social-emotional skills 
were identified as significant intervening or conditioning mechanisms linking IPV exposure and other indicators of well-being (e.g., aggression, psychological distress), thus highlighting social-emotional competence as an important area for intervention. Developmental psychopathology and resilience theory are offered as guiding frameworks for further study of this topic.

\section{Bullying Perpetration and Victimization}

Lee et al. (2021) reviewed 14 articles that examined the relation between IPV exposure and bullying perpetration and victimization. Studies indicated a significant relationship between IPV exposure and both bullying perpetration and victimization. Individual (e.g., gender, race/ethnicity) and family environment (e.g., parenting) characteristics were examined. Multiple practice and policy implications were discussed, including methodological suggestions for future research and potential intervention approaches for IPV-exposed children experiencing bullying perpetration and victimization.

\section{IPV Acceptance and Appraisal}

Evans et al. (2021) reviewed 13 articles that examined children's acceptance of parental IPV and appraisals of IPV, including perceived threat, self-blame attributions, and coping efficacy. A cognitive-contextual theoretical framework guided this review, focusing on how children's adjustment is affected by their perceptions and interpretations of IPV, as well as child-level factors such as child age and gender. Findings revealed that children exposed to IPV had a higher likelihood of accepting parental IPV as normative or justifiable, which in turn predicted youths' ADV perpetration and victimization outcomes. Furthermore, children's appraisals of IPV threat and self-blame served as key cognitive intervening factors to explain the link between IPV exposure and other child outcomes, including self-efficacy, social anxiety, and internalizing problems. Implications for future research are discussed, including the necessity of examining IPV acceptance and appraisals across developmental stages, as well as more focused attention on coping efficacy as a key element in the appraisal process.

\section{Physiological Functioning}

Berg et al. (accepted) reviewed 23 articles that examined the effects of IPV exposure on children's physiological functioning as measured by effects on the hypothalamus-pituitary-adrenal (HPA) axis and both sympathetic and parasympathetic components of the autonomic nervous system
(ANS). Though findings generally demonstrated an effect of IPV exposure on HPA axis dysregulation as indexed by heightened salivary cortisol measured basally and in response to a laboratory stressor, more nuanced measurement of IPV exposure (e.g., recent trajectory of IPV exposure, frequency) will be important in future research delineating patterns of heightened vs. attenuated cortisol reactivity. Children's stress activation response consequent of exposure to IPV was also measured by dysregulation of both sympathetic (indexed by skin conductance level and reactivity, blood pressure, and heart rate) and parasympathetic (indexed by respiratory sinus arrhythmia/vagal tone and reactivity) nervous systems of the ANS. Significant implications are noted for assessment and treatment of IPV-exposed youth, including the necessity of using neurodevelopmentally informed therapeutic approaches that target key bioregulatory functions implicated in the stress response system.

\section{Academic Functioning}

Cage et al. (2021) reviewed 13 articles that examined the effects of IPV exposure on academic functioning. Most reviewed studies identified a negative association between IPV exposure and academic functioning as measured by grade retention, suspension and expulsion, truancy and absenteeism, disciplinary infractions, and failing grades. Negative effects were exacerbated for children dually exposed to IPV and child maltreatment. A primary research implication is the absence of a strong theoretical framework to explain the mechanisms that contribute to differential academic functioning between IPV-exposed and non-exposed youth.

\section{Discussion}

This body of knowledge has grown substantially since the earliest article published by Levine in 1975 describing the detrimental effects of IPV exposure on children. Based on the visual display of the EGM as well as the 135 review studies across the seven papers, we identify below several directions for future research and key implications for practice and policy.

\section{Implications for Research}

In 2005, Margolin published an article describing the six methodological considerations that researchers should use to advance the field of IPV exposure. These recommendations included moving beyond dichotomous group comparisons of children with or without IPV exposure to measuring IPV frequency, severity, and duration; conducting more nuanced assessment involving multiple forms of measurement from 
multiple informants; using comprehensive assessments that consider multiple types of violence exposure; conducting prospective longitudinal research in order to test developmental models; accounting for biological factors that may explain some outcomes; and examining contextual factors to understand the relation between those factors and IPVexposed children's outcomes. Despite the publication of Margolin's article over 15 years ago, many directions for future research illuminated through the reviews in this special section mirror these recommendations. For example, across all seven review articles, authors called attention to an overreliance on parental report of IPV and child outcomes, and a limited number of studies that included measurement of other forms of violence such as child maltreatment. Prioritization of the parent-report Conflict Tactics Scale (CTS; Straus, 1987; CTS2; Straus et al., 2003) to measure child exposure to IPV, and on the physical violence subscale in particular, was also noted across the seven review articles. Parental report of IPV experiences may not be a valid measure of IPV exposure because it does not account for different types of IPV exposure, such as seeing or hearing the violence event, direct involvement such as trying to intervene or experiencing the aftermath of the violence such as observing injuries or mental health symptoms of the survivor (Holden et al., 1998).

Furthermore, all seven of the studies (Bender et al., 2021; Berg et al., accepted; Cage et al., 2021; Evans et al, 2021a, b; Holmes et al., 2021; Lee et al., 2021) identified the need for more longitudinal research, reflecting a recommendation originally articulated by Margolin (2005). As emphasized in each of these studies, longitudinal research is necessary to examine the effects of IPV exposure over time and identify mechanisms linking IPV exposure with a specified biopsychosocial outcome. Longitudinal studies also provide an opportunity to explore moderating factors that may attenuate the relationship between IPV exposure and child health and well-being indicators. Identifying these factors may provide meaningful points of potential intervention to buffer children from the negative sequelae associated with IPV exposure. Furthermore, longitudinal research allows for an exploration of the developmental trajectories of children exposed to IPV, highlighting how these children navigate development within and between different developmental stages (Howell, 2011).

Six of the seven studies in this review (Bender et al., 2021; Berg et al., accepted; Cage et al., 2021; Evans et al., 2021a, b; Holmes et al., 2021) also illuminated the need for developmentally-sensitive research. Children are attending to different tasks at each stage of development, and if exposed to IPV during a particular developmental period, children may experience disruptions in those specific domains (Howell et al., 2016). Research has also suggested that there are certain "developmental windows" when exposure to IPV may have particularly catastrophic effects both in the present and for further developmental trajectories (Howell, 2011; Howell et al., 2016). This makes sense, given what we have come to understand about sequential neurodevelopment; that is, children who experience IPV during early and sensitive developmental windows (e.g., infancy, early childhood) may have compromised neurodevelopment that can shape their adjustment trajectories across the lifespan, and across a variety of developmental domains (Hambrick et al., 2019a, b). Therefore, a developmentallysensitive approach (e.g., measuring the time of IPV exposure, considering neurosequential development, and thinking about outcomes in the context of broader development) is necessary to further our understanding of the effects of IPV exposure on children.

All seven studies (Bender et al., 2021; Berg et al., accepted; Cage et al., 2021; Evans et al., 2021a, b; Holmes et al., 2021; Lee et al., 2021) in this review highlighted the importance of examining and robustly incorporating protective and promotive factors or strengths-focused outcomes into research on children exposed to IPV. This broad review drew attention to a body of research that primarily examines negative outcomes among children exposed to IPV. Very little research to date is assets-focused, resulting in knowledge that is singularly centered around an understanding of which children exhibit (or do not exhibit) negative outcomes. This leaves a devastatingly narrow understanding about which children demonstrate resilience, how they demonstrate resilience, and what internal and external factors promote resilience. Without research that rigorously incorporates protective and promotive factors, there is a limited capacity to develop interventions that promote or support resilience among children exposed to IPV.

The EGM demonstrated the breadth of literature that examined internalizing and externalizing behavior problems among children exposed to IPV, which accounted for over a third of the 381 citations (i.e., a total of 125 studies and 9 review papers). The authors collaboratively decided against synthesizing studies on these outcome domains due to previous reviews already having integrated such literature (e.g., Kitzmann et al., 2003; Vu et al., 2016). The substantial number of studies that examined internalizing and externalizing behavior problems among IPV-exposed children, coupled with the existence of systematic reviews in this area, suggests that future research in this area should examine alternative outcomes or include more nuanced analyses of the subdimensions of internalizing and externalizing behavior problems. This is especially important given the previous discussion on the need to incorporate more strengthsfocused outcomes into research on children exposed to IPV.

Based on EGM, rigorous systematic review papers are needed (none were identified published before August 2020) to synthesize bodies of knowledge among these 
sub-outcomes: delinquency (16 citations), conduct disorder/problems (10 citations), anxiety symptoms/diagnosis (25 citations), peer relationships (13 citations), and verbal/ language ability (9 citations). The EGM also revealed areas of need for expanded research such as animal abuse, addictive behaviors, disordered eating, fear, adjustment, empathy, leadership, non-caregiver relationships, cognitive competence, cognitive coping, and motivation, to name a few.

Aligning with the need for research on protective or promotive factors for more resilient outcomes about children exposed to IPV, the EGM highlighted the need for more research on relational health, or the degree of high quality, attuned, and caring relationships (Hambrick et al., 2019b). Existing research evidences how relational health can buffer children from the negative sequelae associated with trauma exposure. The EGM identified research on parent-child and peer relationship outcomes, but limited research on non-caregiver adult relationship outcomes (e.g., teachers, extended family members) and sibling relationship outcomes. For example, youth developmental asset research has wellestablished the role of natural mentors and supportive nonparental adults in positive socio-emotional and behavioral outcomes among children exposed to a variety of adversities (Bowers et al., 2015; Duke et al., 2017; Pekel et al., 2018). Trusting relationships with non-parental adults can provide youth not only with emotional and instrumental support, but also with a relational template that models safety, empathy, and other essential interpersonal skills (Deutsch et al., 2020). Future research on natural mentors and non-parental supportive adult relationships may offer a promising pathway for non-clinical intervention with IPV-exposed youth, focused on harnessing the potential of naturally-occurring support persons that are already present in their lives. Sibling relationship outcomes, too, warrant further research as existing studies show that siblings relationships can be a source of comfort and safety for IPV-exposed children (Åkerlund, 2017; Berg et al., 2021), and that the quality of these sibling relationships predicts other adjustment outcomes (e.g., internalizing or externalizing behaviors; Iturralde et al., 2013; Piotrowski et al., 2014). Research on how IPV exposure affects sibling relationship quality may further illuminate the role of relational health in predicting child outcomes following IPV exposure.

\section{Implications for Practice and Policy}

Synthesis of findings across this broader systematic review reveals significant themes in the implications for policy and practice. A primary, overarching policy implication is the necessity of infusing a trauma-informed care (TIC) lens (SAMHSA, 2014) into training experiences, agency culture, and supervision for all professionals engaged with this population specifically, and with children in general, including educators, social workers, foster parents, shelter staff, law enforcement, court staff, and medical professionals, among others (Berg et al., accepted; Cage et al., 2021; Holmes et al., 2021). Studies in this review drew samples not only from shelters, but also from communities, schools, mental health agencies, medical care providers, and law enforcement - a range of both nonclinical and clinical settings that are not necessarily designed to screen or treat for IPV exposure. For policymakers, this serves as a reminder that IPV-exposed youth are present in all communities-whether or not they have been screened and identified-and the TIC model ensures that all young people are treated from a traumainformed perspective.

Although TIC models can vary widely in implementation and across settings, the Substance Abuse and Mental Health Services Administration (SAMHSA) has advanced one of the most widely used definitions of trauma-informed care, including a core set of principles to guide TIC service delivery: safety, empowerment, trustworthiness, peer support, cultural sensitivity, and collaboration (Leitch, 2017; Oral et al., 2016; SAMHSA, 2014). Implementation of the TIC model serves to enhance screening and identification of traumatized children and youth, while minimizing risk of retraumatization (Ko et al., 2008; Oral et al., 2016), both of which stand to increase the likelihood of children receiving services and reduce the tremendous economic burden of childhood IPV exposure (Holmes et al., 2018a, b).

In addition to this overarching policy implication for TIC models, several related practice implications are noteworthy, including recommendations around cross-system service engagement, neurodevelopmentally-informed approaches to treatment, and a renewed focus on resilience. Research has demonstrated that comprehensive healing from the trauma of IPV exposure is best facilitated when targeted services are coordinated at all levels of a child's social ecology, including home-, school-, shelter-, medical-, and community-based programming and interventions (Bender et al., 2021; Berg et al., accepted; Cage et al., 2021; Evans et al., 2021a; Holmes et al., 2021; Lee et al., 2021). Cross-system collaboration and coordination is necessary to ensure that intervention and supportive services seamlessly weave across each of these environments. Findings from this review demonstrate the multidimensional impact of IPV exposure on all domains of child functioning, including social-emotional, physical, cognitive, and behavioral health. Outcomes specific to each of these domains can present differently depending on a child's setting; consequently, interventions designed to optimize child functioning in each setting offer the most holistic and intensive approach to treatment. While cross-system coordination can be challenging, there is evidence that such models are both feasible and effective with general child maltreatment populations, as demonstrated by the Massachusetts Child Trauma Project (Bartlett et al., 2016) and the 
Safe Start Demonstration Projects (Arteaga \& Lamb, 2008; Hyde et al, 2008). Such multilevel intervention programs highlight a future direction for comprehensive IPV exposure treatment models.

A second key practice implication drawn from this review is the importance of recognizing the role that dysregulated stress response systems may play in children's maladaptive outcomes (Berg et al., accepted). Each of the outcome categories covered in this review taps into the domains of child impairment affected by complex trauma: attachment, biology, affect regulation, dissociation, behavioral regulation, cognition, and self-concept (Cook et al., 2017; van der Kolk, 2017). Among complexly traumatized children, maladaptation in these domains can often be traced to neurodevelopmental changes consequent of chronic activation of the body's stress response system, particularly in early childhood when the brain is rapidly organizing and developing (Gaskill \& Perry, 2012). Prolonged activation of the stress response system during vulnerable developmental windows can disrupt a child's developing capacity for self-regulation, stress coping, and cognitive processing (Herringa, 2017).

This review highlights the ways that such neurodevelopmental disruptions can unfold across settings and functional domains for children, such as poor academic performance (e.g., impaired cognitive processing or attentional capacity, dissociation; Cage et al., 2021), bullying or dating violence perpetration (e.g., aggression/affect and behavioral dysregulation; Evans et al., 2021a; Lee et al., 2021), self-blaming appraisals of IPV (e.g., self-concept; Evans et al., 2021b), poor health outcomes (e.g., compromised lung functioning; Holmes et al., 2021), and underdeveloped social-emotional skills (e.g., self-regulation; Bender et al., 2021). Recognizing the potential role of altered neurodevelopment in IPV-exposure symptomatology can help shape practitioners' treatment sequence. For example, neurodevelopmental approaches to treatment emphasize the importance of first helping a child develop basic self-regulatory capacities, alongside the consistent care of trusting and supportive adults, before progressing to cognitive aspects of treatment that require reasoning and decision-making skills (Perry, 2009). Importantly, promising neurodevelopmental approaches can be incorporated into a variety of settings and can be integrated to existing service programs (Barfield et al., 2012; de Nooyer \& Lingard, 2017; Ryan et al., 2017).

Finally, this EGM points to the tremendous degree of resilience and positive adaptation evidenced in children exposed to IPV, which has powerful implications for practitioners. Despite the vulnerabilities and risks posed to this group of young people, the studies reviewed consistently demonstrate that many children display adaptive biopsychosocial functioning, achieve appropriate developmental milestones, and navigate their social environments with great fluency and competence (Bender et al., 2021; Cage et al., 2021; Evans et al., 2021b). For practitioners, this highlights the importance of building on the strengths and assets IPV-exposed youth already possess in order to further enhance their coping skills and development. Interventions that foster additional supports associated with resilience in IPV-exposed youth should be prioritized, including involvement with prosocial peers, school supports, and family-level resilience factors such as maternal warmth, maternal mental health, and positive parenting practices (Fogarty et al., 2019; Yule et al., 2019). Interventions that foster relational health, for example those that support higher-quality family or peer relationships, also warrant prioritization (e.g., Hambrick et al., 2019b). Furthermore, we must be cautious to not assume that resilience at one stage of development suggests IPV-exposed children do not need, or benefit less from, intervention. For example, sleeper effects have been demonstrated in young IPV-exposed children, suggesting that behavioral symptoms (e.g., aggression) may manifest in later developmental stages even among IPV-exposed children who initially indicate adaptive functioning (Holmes, 2013).

\section{Conclusion}

This special section rigorously synthesized existing literature on the effects of IPV exposure on child outcomes associated with medical system engagement and physical health outcomes, ADV victimization and perpetration, social-emotional competence, bullying victimization and perpetration, IPV appraisal and acceptance, physiological functioning, and academic functioning. This consolidation of empirical research also meaningfully informs future directions for research and implications for policy and practice in each specific domain and in the broader scope of our understanding of child exposure to IPV. Our hope is that these findings will contribute to further advancements in knowledge of the effects of IPV exposure on children and more innovative policies and practices that prioritize trauma-informed approaches to better serve this population.

Funding Funded through The HealthPath Foundation of Ohio. The contents of this publication do not necessarily reflect the views or policies of the funders. This information is in the public domain. Readers are encouraged to copy and share it, but please credit the authors.

\section{References}

Achenbach, T. M., \& Rescorla, L. A. (2001). Manual for the ASEBA school-age forms \& profiles. University of Vermont, Research Center for Children, Youth, \& Families.

Åkerlund, N. (2017). Caring or vulnerable children? Sibling relationships when exposed to intimate partner violence. Children \& Society, 31(6), 475-485. https://doi.org/10.1111/chso.12215 
Arteaga, S. S., \& Lamb, Y. (2008). Expert review of key findings on children exposed to violence and their families from the Safe Start Demonstration Project. Best Practices in Mental Health, 4(1), 99-107.

Barfield, S., Dobson, C., Gaskill, R., \& Perry, B. D. (2012). Neurosequential model of therapeutics in a therapeutic preschool: Implications for work with children with complex neuropsychiatric problems. International Journal of Play Therapy, 21(1), 30-44. https://doi.org/10.1037/a0025955

Bartlett, J. D., Barto, B., Griffin, J. L., Fraser, J. G., Hodgdon, H., \& Bodian, R. (2016). Trauma-informed care in the Massachusetts Child Trauma Project. Child Maltreatment, 21(2), 101-112. https://doi.org/10.1177/1077559515615700

*Bender, A. E., McKinney, S. J., Schmidt-Sane, M. M., Cage, J., Holmes, M. R., Berg, K. A., Salley, J., Bodell, M, Miller, E. K., \& Voith, L. A. (2021). Childhood exposure to intimate partner violence and effects on social-emotional competence: A systematic review. Journal of Family Violence. https://doi.org/10.1007/ s10896-021-00315-Z

Berg, K. A., Francis, M. W., Ross, K., \& Spilsbury, J. C. (2021). Opportunities to improve sleep of children exposed to interpersonal violence: A social-ecological perspective. Children and Youth Services Review, 127, 106082. https://doi.org/10.1016/j. childyouth.2021.106082

*Berg, K. A., Evans, K. E., Powers, G., Moore, S. E., Steigerwald, S., Bender, A. E., Holmes, M. R., Yaffe, A., \& Connell, A. M. (Accepted pending minor revisions: JOFV-D-21-00252). Exposure to intimate partner violence and children's physiological functioning: A systematic review of the literature.

Bowers, E. P., Johnson, S. K., Warren, D. J., Tirrell, J. M., \& Lerner, J. V. (2015). Youth-adult relationships and positive youth development. In Promoting positive youth development (pp. 97-120). Springer, Cham.

*Cage, J., Kobulsky, J. M., McKinney, S. J., Holmes, M. R., Berg, K. A., Bender, A. E., \& Kemmerer, A. (2021). The effect of exposure to intimate partner violence on children's academic functioning: A systematic review of the literature. Journal of Family Violence. https://doi.org/10.1007/s10896-021-00314-0

Cook, A., Spinazzola, J., Ford, J., Lanktree, C., Blaustein, M., Cloitre, M., deRosa, R., Hubbard, R., Kagan, R., Liautaud, J., Mallah, K., Olafson, E., \& van der Kolk, B. (2017). Complex trauma in children and adolescents. Psychiatric Annals, 35(5), 390-398. https:// doi.org/10.3928/00485713-20050501-05

Davis, L. V., \& Carlson, B. E. (1987). Observation of spouse abuse: What happens to the children? Journal of Interpersonal Violence, 2(3), 278-291. https://doi.org/10.1177/088626087002003004

de Nooyer, K. M., \& Lingard, M. W. (2017). Applying principles of the neurosequential model of therapeutics across an adolescent day program and inpatient unit. Australasian Psychiatry, 25(2), 150-153. https://doi.org/10.1177/1039856216658824

Deutsch, N. L., Mauer, V. A., Johnson, H. E., Grabowska, A. A., \& Arbeit, M. R. (2020). "[My counselor] knows stuff about me, but [my natural mentor] actually knows me": Distinguishing characteristics of youth's natural mentoring relationships. Children and Youth Services Review, 111, 104879. https://doi.org/10.1016/j. childyouth.2020.104879

Duke, T., Farruggia, S. P., \& Germo, G. R. (2017). "I don't know where I would be right now if it wasn't for them": Emancipated foster care youth and their important non-parental adults. Children and Youth Services Review, 76, 65-73. https://doi.org/10.1016/j.child youth.2017.02.015

Edleson, J. L. (1999). Children's witnessing of adult domestic violence. Journal of Interpersonal Violence, 14(8), 839-870. https://doi. org/10.1177/088626099014008004

Edleson, J. L., Shin, N., \& Armendariz, K. K. J. (2008). Measuring children's exposure to domestic violence: The development and testing of the Child Exposure to Domestic Violence (CEDV) Scale. Children and Youth Services Review, 30(5), 502-521. https://doi.org/10.1016/j.childyouth.2007.11.006

Evans, S. E., Davies, C., \& DiLillo, D. (2008). Exposure to domestic violence: A meta-analysis of child and adolescent outcomes. Aggression and Violent Behavior, 13(2), 131-140. https://doi.org/ 10.1016/j.avb.2008.02.005

*Evans, K. E., Lee, H., Russell, K. N., Holmes, M. R., Berg, K. A., Bender, A. E., \& Prince, D. M. (2021a). Adolescent dating violence among youth exposed to intimate partner violence: A systematic review. Journal of Family Violence. https://doi.org/10. 1007/s10896-021-00289-y

*Evans, K. E., Schmidt-Sane, M. M., Bender, A. E., Berg, K. A., \& Holmes, M. R. (2021b). Children's exposure to intimate partner violence and acceptance or appraisals of IPV: A systematic review. Journal of Family Violence. https://doi.org/10.1007/ s10896-021-00318-w

Fantuzzo, J. W., \& Lindquist, C. U. (1989). The effects of observing conjugal violence on children: A review and analysis of research methodology. Journal of Family Violence, 4(1), 77-94. https:// doi.org/10.2307/1602779

Fantuzzo, J. W., \& Mohr, W. K. (1999). Prevalence and effects of child exposure to domestic violence. The Future of Children, 9(3), 21-32. https://www.jstor.org/stable/1602779.

Fogarty, A., Wood, C. E., Giallo, R., Kaufman, J., \& Hansen, M. (2019). Factors promoting emotional-behavioural resilience and adjustment in children exposed to intimate partner violence: A systematic review. Australian Journal of Psychology, 71(4), 375389. https://doi.org/10.1111/ajpy. 12242

Fortin, A., Doucet, M., \& Damant, D. (2011). Children's appraisals as mediators of the relationship between domestic violence and child adjustment. Violence and Victims, 26(3), 377-392. https://doi.org/ 10.1891/0886-6708.26.3.377

Gaskill, R. L., \& Perry, B. D. (2012). Child sexual abuse, traumatic experiences, and their impact on the developing brain. In P. GoodYear Brown (Ed.), Handbook of child sexual abuse: Identification, assessment, and treatment (pp. 29-47). Wiley.

Gewirtz, A. H., \& Edleson, J. L. (2007). Young children's exposure to intimate partner violence: Towards a developmental risk and resilience framework for research and intervention. Journal of Family Violence, 22(3), 151-163. https://doi.org/10.1007/ s10896-007-9065-3

Graham-Bermann, S. A., \& Hughes, H. M. (1999). The impact of domestic violence and emotional abuse on children: The intersection of research, theory, and clinical intervention. Journal of Emotional Abuse, 1(2), 1-21. https://doi.org/10.1300/J135v01n02_01

Graham-Bermann, S. A., Gruber, G., Howell, K. H., \& Girz, L. (2009). Factors discriminating among profiles of resilience and psychopathology in children exposed to intimate partner violence (IPV). Child Abuse \& Neglect, 33(9), 648-660. https://doi.org/10.1016/j. chiabu.2009.01.002

Hambrick, E. P., Brawner, T. W., \& Perry, B. D. (2019a). Timing of early-life stress and the development of brain-related capacities. Frontiers in Behavioral Neuroscience, 13(183), 1-14. https://doi. org/10.3389/fnbeh.2019.00183

Hambrick, E. P., Brawner, T. W., Perry, B. D., Brandt, K., Hofmeister, C., \& Collins, J. O. (2019b). Beyond the ACE score: Examining relationships between timing of developmental adversity, relational health and developmental outcomes in children. Archives of Psychiatric Nursing, 33(3), 238-247. https://doi.org/10.1016/j. apnu.2018.11.001

Herringa, R. J. (2017). Trauma, PTSD, and the developing brain. Current Psychiatry Reports, 19(69), 1-9. https://doi.org/10.1007/ s11920-017-0825-3

Holden, G. W., Geffner, R. E., \& Jouriles, E. N. (Eds.). (1998). APA science volumes. Children exposed to marital violence: 
Theory, research, and applied issues. American Psychological Association.

Holmes, M. R. (2013). The sleeper effect of intimate partner violence exposure: Long-term consequences on young children's aggressive behavior. Journal of Child Psychology and Psychiatry, 54(9), 986-995. https://doi.org/10.1111/jcpp.12071

Holmes, M. R., Richter, F. G., Votruba, M. E., Berg, K. A., \& Bender, A. E. (2018a). Economic burden of child exposure to intimate partner violence in the United States. Journal of Family Violence, 33(4), 239-249. https://doi.org/10.1007/s10896-018-9954-7

Holmes, M. R., Yoon, S., Berg, K., Cage, J., \& Perzynski, A. T. (2018b). Promoting the development of resilient academic functioning in maltreated children. Child Abuse \& Neglect, 75, 92-103. https://doi.org/10.1016/j.chiabu.2017.07.018

*Holmes, M. R., Berg, K. A., Bender, A. E., Evans, K. E., Kobulsky, J. M., Davis, A. P. \& King, J. A. (2021). The effect of intimate partner violence on children's medical system engagement and physical health: A systematic review. Journal of Family Violence. https://doi.org/10.1007/s10896-021-00291-4

Holt, S., Buckley, H., \& Whelan, S. (2008). The impact of exposure to domestic violence on children and young people: A review of the literature. Child Abuse \& Neglect, 32(8), 797-810. https://doi.org/ 10.1016/j.chiabu.2008.02.004

Howell, K. H. (2011). Resilience and psychopathology in children exposed to family violence. Aggression and Violent Behavior, 16(6), 562-569. https://doi.org/10.1016/j.avb.2011.09.001

Howell, K. H., Barnes, S. E., Miller, L. E., \& Graham-Bermann, S. A. (2016). Developmental variations in the impact of intimate partner violence exposure during childhood. Journal of Injury and Violence Research, 8(1), 1-15. https://doi.org/10.5249/jivr. v8i1.663

Huang, C. C., Wang, L. R., \& Warrener, C. (2010). Effects of domestic violence on behavior problems of preschool-aged children: Do maternal mental health and parenting mediate the effects? Children and Youth Services Review, 32(10), 1317-1323. https://doi. org/10.1016/j.childyouth.2010.04.024

Hughes, H. M., \& Barad, S. J. (1983). Psychological functioning of children in a battered women's shelter: A preliminary investigation. American Journal of Orthopsychiatry, 53(3), 525-531. https://doi.org/10.1111/j.1939-0025.1983.tb03396.x

Hughes, H. M., Graham-Bermann, S. A., \& Gruber, G. (2001). Resilience in children exposed to domestic violence. In S. A. GrahamBermann \& J. L. Edleson (Eds.), Domestic violence in the lives of children: The future of research, intervention, and social policy (pp. 67-90). American Psychological Association.

Hyde, M. M., Lamb, Y., Arteaga, S. S., \& Chavis, D. (2008). National evaluation of the Safe Start Demonstration Project: Implications for mental health practice. Best Practice in Mental Health, 4(1), $108-122$

Iturralde, E., Margolin, G., \& Spies Shapiro, L. A. (2013). Positive and negative interactions observed between siblings: Moderating effects for children exposed to parents' conflict. Journal of Research on Adolescence, 23(4), 716-729. https://doi.org/10. 1111/jora. 12020

Katz, D. L., Williams, A. L., Girard, C., \& Goodman, J. (2003). The evidence base for complementary and alternative medicine: Methods of evidence mapping with application to CAM. Alternative Therapies in Health and Medicine, 9(4), 22-30.

Kitzmann, K. M., Gaylord, N. K., Holt, A. R., \& Kenny, E. D. (2003). Child witnesses to domestic violence: A meta-analytic review. Journal of Consulting and Clinical Psychology, 71(2), 339-352. https://doi.org/10.1037/0022-006X.71.2.339

Ko, S. J., Ford, J. D., Kassam-Adams, N., Berkowitz, S. J., Wilson, C., Wong, M., Brymer, M. J., \& Layne, C. M. (2008). Creating trauma-informed systems: Child welfare, education, first responders, health care, juvenile justice. Professional Psychology:
Research and Practice, 39(4), 396-404. https://doi.org/10.1037/ 0735-7028.39.4.396

*Lee, H., Russell, K. N., O’Donnell, K. A., Miller, E. K., Bender, A. E., Scaggs, A. L., Harris, L. A., III., Holmes, M. R., \& Berg, K. A. (2021). The effect of childhood intimate partner violence (IPV) exposure on bullying: A systematic review. Journal of Family Violence. https://doi.org/10.1007/s10896-021-00299-w

Leitch, L. (2017). Action steps using ACEs and trauma-informed care: A resilience model. Health \& Justice, 5(1), 5-15. https://doi.org/ 10.1186/s40352-017-0050-5

Levine, M. D. (1975). Interpersonal violence and its effects on the children: A study of 50 families in general practice. Medicine, Science and Law, 15, 172-176.

Lichter, E. L., \& McCloskey, L. A. (2004). The effects of childhood exposure to marital violence on adolescent gender-role beliefs and dating violence. Psychology of Women Quarterly, 28(4), 344-357. https://doi.org/10.1111/j.1471-6402.2004.00151.x

Margolin, G. (1998). Effects of domestic violence on children. In P. K. Trickett \& C. J. Schellenbach (Eds.), Violence against children in the family and the community (pp. 57-101). American Psychological Association.

Margolin, G. (2005). Children's exposure to violence: Exploring developmental pathways to diverse outcomes. Journal of Interpersonal Violence, 20(1), 72-81. https://doi.org/10.1177/08862 60504268371

Martinez-Torteya, C., Anne Bogat, G., Von Eye, A., \& Levendosky, A. A. (2009). Resilience among children exposed to domestic violence: The role of risk and protective factors. Child Development, 80(2), 562-577. https://doi.org/10.1111/j.1467-8624. 2009.01279.x

O’ Keefe, M. (1994). Adjustment of children from maritally violent homes. Families in Society, 75(7), 403-415. https://doi.org/10. 1177/104438949407500701

Oral, R., Ramirez, M., Coohey, C., Nakada, S., Walz, A., Kuntz, A., Benoit, J., \& Peek-Asa, C. (2016). Adverse childhood experiences and trauma informed care: The future of healthcare. Pediatric Research, 79(1), 227-233. https://doi.org/10.1038/ pr.2015.197

Osofsky, J. D. (1998). Children as invisible victims of domestic and community violence. In G. W. Holden, R. Geffner, \& E. N. Jouriles (Eds.), Children exposed to marital violence: Theory, research and applied issues (pp. 95-117). American Psychological Association.

Owen, A. E., Thompson, M. P., \& Kaslow, N. J. (2006). The mediating role of parenting stress in the relation between intimate partner violence and child adjustment. Journal of Family Psychology, 20(3), 505-513. https://doi.org/10.1037/0893-3200. 20.3.505

Pekel, K., Roehlkepartain, E. C., Syvertsen, A. K., Scales, P. C., Sullivan, T. K., \& Sethi, J. (2018). Finding the fluoride: Examining how and why developmental relationships are the active ingredient in interventions that work. American Journal of Orthopsychiatry, 88(5), 493-502.

Perry, B. D. (2009). Examining child maltreatment through a neurodevelopmental lens: Clinical applications of the neurosequential model of therapeutics. Journal of Loss and Trauma, 14(4), 240-255.

Piotrowski, C. C., Tailor, K., \& Cormier, D. C. (2014). Siblings exposed to intimate partner violence: Linking sibling relationship quality \& child adjustment problems. Child Abuse \& Neglect, 38(1), 123-134. https://doi.org/10.1016/j.chiabu.2013.08.005

Ravi, K. E., \& Tonui, B. C. (2020). A systematic review of the Child Exposure to Domestic Violence scale. The British Journal of Social Work, 50(1), 101-118. https://doi.org/10.1093/bjsw/bcz028

Rosenbaum, A., \& O'Leary, K. D. (1981). Children: The unintended victims of marital violence. American Journal of Orthopsychiatry, 
51(4), 692-699. https://doi.org/10.1111/j.1939-0025.1981.tb014 16.x

Ryan, K., Lane, S. J., \& Powers, D. (2017). A multidisciplinary model for treating complex trauma in early childhood. International Journal of Play Therapy, 26(2), 111-123. https://doi.org/10.1037/ pla0000044

Saran, A., \& White, H. (2018). Evidence and gap maps: A comparison of different approaches. Campbell Systematic Reviews, 14(1), 1-38. https://doi.org/10.4073/cmdp.2018.2

Stagg, V., Wills, G. D., \& Howell, M. (1989). Psychopathology in early childhood witnesses of family violence. Topics in Early Childhood Special Education, 9(2), 73-87. https://doi.org/10.1177/ 027112148900900206

Straus, M. A., Hamby, S. L., \& Warren, W. L. (2003). The Conflict Tactics Scales handbook: Revised Conflict Tactics Scales (CTS2): CTS: Parent-Child Version (CTSPC). Western Psychological Services.

Straus, M. A. (1987). The Conflict Tactics Scales and its critics: An evaluation and new data on validity and reliability. New Hampshire University, Family Research Lab. ERIC Clearinghouse. Retrieved from https://eric.ed.gov/?id=ED297030

Substance Abuse and Mental Health Services Administration. (2014). SAMHSA's concept of trauma and guidance for a trauma-informed approach. HHS Publication No. (SMA) 14-4884. Substance Abuse and Mental Health Services Administration.

van der Kolk, B. A. (2017). Developmental trauma disorder: Toward a rational diagnosis for children with complex trauma histories. Psychiatric Annals, 35(5), 401-408. https://doi.org/10.13109/ prkk.2009.58.8.572

Vu, N. L., Jouriles, E. N., McDonald, R., \& Rosenfield, D. (2016). Children's exposure to intimate partner violence: A meta-analysis of longitudinal associations with child adjustment problems. Clinical Psychology Review, 46, 25-33. https://doi.org/10.1016/j. cpr.2016.04.003

Wolfe, D. A., Crooks, C. V., Lee, V., McIntyre-Smith, A., \& Jaffe, P. G. (2003). The effects of children's exposure to domestic violence: A meta-analysis and critique. Clinical Child and Family Psychology Review, 6(3), 171-187. https://doi.org/10.1023/A:1024910416164

Yule, K., Houston, J., \& Grych, J. (2019). Resilience in children exposed to violence: A meta-analysis of protective factors across ecological contexts. Clinical Child and Family Psychology Review, 22(3), 406-431. https://doi.org/10.1007/ s10567-019-00293-1

Publisher's Note Springer Nature remains neutral with regard to jurisdictional claims in published maps and institutional affiliations. 